\title{
Echovirus 18 Infection Is Associated with Crohn's Disease
}

\author{
John Chia, Andrew Chia, David Wang, Rabiha El-Habbal, Sitaraman Jyotheeswaran, Eric McCloud \\ ${ }^{1}$ EV Med Research, Lomita, CA, USA \\ ${ }^{2}$ Adult Gastroenterology, Torrance, CA, USA \\ ${ }^{3}$ Pediatric Gastroenterology, Torrance, CA, USA \\ Email: *evmedresearch@gmail.com
}

How to cite this paper: Chia, J., Chia, A., Wang, D., El-Habbal, R., Jyotheeswaran, S. and McCloud, E. (2019) Echovirus 18 Infection Is Associated with Crohn's Disease. Open Journal of Gastroenterology, 9, 174-183. https://doi.org/10.4236/ojgas.2019.98020

Received: July 29, 2019

Accepted: August 20, 2019

Published: August 23, 2019

Copyright $\odot 2019$ by author(s) and Scientific Research Publishing Inc. This work is licensed under the Creative Commons Attribution International License (CC BY 4.0).

http://creativecommons.org/licenses/by/4.0/

\begin{abstract}
Background: Enterovirus (EV) can cause gastroenteritis and are known to replicate in Peyer's patches of terminal ileum. EV has been found in the intestinal specimens from immunocompromised patients with regional enteritis, and another study demonstrates the presence of enterovirus in the resected terminal ileum of immunocompetent pediatric patients with Crohn's disease (CD). Cluster outbreaks of CD have also been reported in the literature but the cause of the disease remains elusive. Materials and Methods: A small cluster of pathologically proven CD occurred in our geographic area in 2004-2005, concurrently with an epidemic of Echovirus 18 (E18) meningitis. Serum samples of these and other $\mathrm{CD}$ patients and control subjects were tested for neutralizing antibodies of 11 common typeable enteroviruses and Echovirus 18; and tissue samples of CD patients, and terminal ileum and colon biopsies of normal controls were tested for the presence of viral capsid protein 1 (VP1) by immunoperoxidase staining. Results: Immunoperoxidase staining demonstrated VP1 in a rare epithelial granuloma, in diseased muscle of terminal ileum and also in colon biopsies of CD patients. Significantly elevated E18 neutralizing antibody was found in patients with pathologically-proven Crohn's disease, as compared to control subjects. Conclusion: In this small observational study, EV VP1 is consistently demonstrated in tissue samples of CD patients as compared to control subjects; and neutralizing antibody for E18 was found in all of the patients with available serum samples. Larger cross-sectional studies will be needed to define the role of E18 in this chronic disease.
\end{abstract}

\section{Keywords}

Enterovirus, Crohn's Disease, Neutralizing Antibody, Immunoperoxidase Staining 


\section{Introduction}

Enteroviruses (EV) are common causes of gastrointestinal, respiratory and non-specific flu-like illnesses, followed by disseminated infections in some cases. Many cases may be asymptomatic. Based on a 25 -year surveillance study, published by the Centers for Diseases Control (CDC), it is estimated as many as 10 15 million of the U.S. population would develop symptomatic infection annually [1]. However, most infectious diseases specialists and gastroenterologists are not familiar with these infections, and even modern stool PCR test does not routinely test for these viruses.

Enteroviruses are known to replicate in Peyer's patch of terminal ileum, a gut-associated lymphoid tissue. The importance of Peyer's patches in the development of Crohn's disease (CD) is well-accepted, and EV has been reported to cause regional enteritis in a few patients with agammaglobulinemia [2] [3] [4] [5]. Recently, Nyström et al. documented the presence of enterovirus in the resected terminal ileum of immunocompetent pediatric patients with Crohn's disease [6]. Coxsackieviruses and echoviruses were both implicated but no specific serotype was diagnosed in these cases.

Echovirus 18 (E18) has been reported to cause epidemics of meningitis and gastroenteritis in the last 25 years; the major outbreaks were between years 1986-1987 and 1995-2005 [1]. In our local area, E18 was found in many CSF samples of patients with viral meningitis between 2004 and 2005 by viral capsid protein 1 (VP1) gene sequencing, using a previously described method [7]; at the same time we observed small cluster outbreaks of Crohn's disease in adults and pediatric patients $(n=4)$. We asked the question of whether E18 could be associated with Crohn's disease.

\section{Materials and Methods}

Patients with clinical and pathologic diagnosis of $\mathrm{CD}$ were evaluated by at least one of the physician authors: 4 patients during the outbreak, 2 other patients who had known Crohn's disease acquired 20 years earlier and 4 patients diagnosed in 2010-2012 were included in the study (Table 1). Patients gave consent to obtain serum and archived tissue samples for viral antibody and immunochemical studies, respectively. Normal patients with prior screening colonoscopies and random biopsies of the colon/terminal ileum were enrolled as control subjects $(n=78)$. In addition, patients seen in clinic for the diagnosis of myalgic encephalomyelitis/chronic fatigue syndrome in the same periods were screened for the presence of E18 neutralizing antibody (n-85) as control subjects. The study was approved by the Institution Review Board of Providence Little Company of Mary Hospital, Torrance CA.

Immunoperoxidase staining. Immunoperoxidase staining of the paraffin-embedded intestinal tissues was performed with an enterovirus-specific murine monoclonal antibody $(\mathrm{mAb})$ directed against a highly conserved viral capsid protein (VP1) sequence (5D8/1 at 1:2000 dilutions, Dako, Carpenteria, CA) 
Table 1. Age, disease duration, antibody titers for EV and EV protein staining of tissues.

\begin{tabular}{|c|c|c|c|c|c|c|c|c|c|c|c|c|c|c|c|c|c|}
\hline$\#$ & age & year & echo 18 & CVB1 & CVB2 & CVB3 & CVB4 & CVB5 & CVB6 & Ech6 & Ech7 & Ech9 & Ech11 & Ech30 & $\begin{array}{l}\text { Colon } \\
\text { epith. }\end{array}$ & $\begin{array}{c}\text { TI } \\
\text { epith. }\end{array}$ & TI muscle \\
\hline 1 & 16 & 0.1 & $>80$ & $<80$ & $<80$ & $<80$ & $<80$ & $<80$ & nd & $<80$ & $<80$ & nd & $<80$ & nd & + & - & nd \\
\hline 2 & 16 & 0.1 & $>80$ & $<80$ & $<80$ & $<80$ & $<80$ & $<80$ & nd & $<80$ & $<80$ & nd & $<80$ & $\mathrm{Nd}$ & + & - & nd \\
\hline 3 & 14 & 0.3 & nd & 5 & 20 & 80 & 20 & 5 & 5 & 20 & 40 & 80 & 5 & 5 & - & + & nd \\
\hline 4 & 55 & 0.2 & $>80$ & 5 & 5 & 40 & 10 & 5 & 5 & 5 & 5 & 5 & 5 & 5 & - & - & + \\
\hline 5 & 70 & 20.0 & $>80$ & 80 & 40 & 5 & 640 & 5 & 5 & 20 & 5 & 5 & 5 & 5 & + & - & + \\
\hline 6 & 56 & 20.0 & $>80$ & nd & nd & nd & nd & nd & nd & nd & nd & nd & nd & nd & + & - & + \\
\hline 7 & 18 & 1.0 & $>80$ & $<80$ & $<80$ & $<80$ & $<80$ & $<80$ & nd & $<80$ & $<80$ & nd & $>80$ & nd & + & - & nd \\
\hline 8 & 21 & 2.0 & $>80$ & $<80$ & $>80$ & $>80$ & $<80$ & $<80$ & nd & $<80$ & $<80$ & nd & $<80$ & nd & + & - & nd \\
\hline 9 & 25 & 1.0 & $>80$ & $<80$ & $<80$ & $<80$ & $<80$ & $<80$ & nd & $<80$ & $<80$ & nd & $<80$ & nd & + & - & nd \\
\hline 10 & 26 & 3.0 & nd & nd & nd & nd & nd & nd & nd & nd & nd & nd & nd & nd & + & - & + \\
\hline
\end{tabular}

Bolded age represents female gender. Duration of disease represents time of serum sampling in respect to disease onset. Shaded rows represent antibody titers performed at ARUP laboratory, Salt lake city, Utah. Negative titer was $<1: 10$ and a titer of $\geq 1: 80$ was considered as evidence of past or current infection. The rest of serum samples were screened by in-house method at 1:80 dilutions. Nd-not done.

or a CMV mAb with the same isotype (Chemicon, Temecula, CA, 1:2000 dilutions) as negative control, following by staining protocol as described previously except two hydrogen peroxide incubation steps were performed to remove endogenous peroxidase activity of the muscle [8] [9] [10]. Hematoxylyn was used as counter stain. Selected samples were first pre-incubated with 1:100 dilutions of polyclonal rabbit anti-creatine kinase B and polyclonal anti-ATPase (Abcam, Cambridge, Ma) for 30 minutes before adding 5D8/1 to block non-specific binding to cross-reactive human creatine kinase B (CKb) and ATPase [11] [12] [13] [14]. All the staining runs were done manually since the use of semi-automated stainer resulted in unacceptable background staining even with more dilution of the primary antibody.

Cell lines and growth condition. Buffalo green monkey kidney cells-with human decay accelerating factor (BGMK-DAF cells) and $\mathrm{CaCO}_{2}$ cells were purchased from Diagnostic Hybrid (Athens, Oh), and American Tissue Culture Company (ATCC, Manassas, VA), respectively. Cells were grown in advance minimal essential media (AMEM, life technology, Grand Island, NY) supplemented with $2 \%$ fetal calf serum at $37^{\circ} \mathrm{C}$ with $5 \% \mathrm{CO}_{2}$.

Neutralizing antibody assay. For 3 patients, antibody titers to Coxsackievirus $B 1,2,3,4,5,6$ and echovirus $6,7,9,11,30$ by micro-neutralization assay were performed by ARUP laboratory, Salt lake city, Utah. Negative titer was $<1: 10$ and a titer of $\geq 1: 80$ was considered as evidence of past or current infection.

In-house neutralization assay: For most of the serum samples, a modified in-house screening neutralization assay was used to determine titers of neutralizing antibody for the same 11 viruses. CVB1-6, echoviruses 6, 7, 9, 11, 30 were purchased from ATCC. Approximately $50 \mu \mathrm{L}$ of $4 \times$ quantity of viruses, consistently caused complete lysis of BGMK-DAF cells in $48-72$ hours, was mixed with $50 \mu \mathrm{L}$ of 1:40 dilution of the serum samples for one hour at room tempera- 
ture before adding to confluent monolayer of BGMK-DAF cells in 24-well plate in duplicates. In 48 - 72 hours the cells were fixed with $4 \%$ formaldehyde, washed and stained with crystal violet. Neutralization is defined as complete protection of the monolayer, and the antibody titers was expressed as $<$ or $\geq 1: 80$ for negative or positive neutralization, respectively. For viruses that did not lyse the monolayers during repeated assay, the results were reported as not done (ND).

E18 neutralization assay. CSF isolates of E18 from patients with viral meningitis from the same periods as the Crohn's diseases outbreak were genotyped in 2012, using VP1 RT-PCR and sequencing as described by Oberste et al. [7]. Although high M.O.I. of E18 could lyse BGMK-DAF and $\mathrm{CaCO}_{2}$ cells within one week of virus inoculation, much smaller inoculum did not result in lysis or plaque formation in either cell monolayer for over 10 - 14 days; and growth was much slower in $\mathrm{CaCO}_{2}$ cells. A modified protocol was used to quantitate enterovirus RNA within the infected cells in 48 hours after infection. Viruses equivalent to 5000 - 7500 copies of EV RNA were pre-incubated with and without 1:40 dilution of serum samples for $1 / 2$ hour before adding to the monolayer of $\mathrm{CaCO}_{2}$ cells for 2 hours. The infected monolayers were washed with Hank balanced salt solution twice and refed with media. In 48 hours, the monolayers of duplicate wells were washed, lysed in RLT buffer, and the RNA of the pooled lysates extracted using the RNeasy mini kit (Qiagen, Valencia, Ca). Enterovirus RNA was quantitated using Pan-enterovirus Amplifluor primers kit with cDNA standard (Millipore, catalog \# AMP3100), Qiagen one-step RT-PCR enzyme kit and Step-one plus real-time PCR thermocycler (Applied Biosystem, Palo Alto, Ca). 2 $\log _{10}$ reduction of viral RNA in the wells treated with antibody comparing to wells with no added antibody was considered as evidence for neutralization at a titer of $\geq 1: 80$.

Enteroviral RNA testing of the tissue specimens: Four $10 \mu \mathrm{M}$ paraffin sections of the intestine tissue specimens from $C D$ patients and controls were processed with PureLink FFPE RNA isolation kit (Invitrogen, Carlsbad, CA, USA), according to the manufacturer's instructions. Extracted RNA was amplified for enterovirus RNA as described above [8].

Case studies during the cluster outbreak in 2004-2005: Patients 1, 2: Within one week a pair of 16 year-old identical twin males was admitted to hospital for severe RLQ pain and bloody diarrhea. Biopsies of ileum and colon showed chronic inflammatory changes including cryptitis. Within one month after discharge, both patients developed intestinal obstruction resulting in partial resection of terminal ileum at a different hospital. The pathology was consistent with Crohn's disease but specimens were not available for staining. Patient 3:14 year-old white female developed bloody diarrhea and right lower quadrant (RLQ) abdominal pain. CT scan of the abdomen and pelvis demonstrated inflammation of the terminal ileum and cecum; colonoscopic examination showed inflammation in focal areas of colon and terminal ileum (TI). She developed recurrent TI obstruction but avoided surgery over the next 8 years. Patient 4: 55 
y/o white male developed small bowel obstruction 6 months after developing RLQ pain. The patient underwent partial resection of TI and cecum. The pathological finding was consistent with severe Crohn' disease. The patient characteristics are summarized in Table 1.

\section{Result of Immunochemical Studies}

For case 3, immunoperoxidase staining with $5 \mathrm{D} 8 / 1$ demonstrated viral protein in a rarely found granuloma of TI biopsy (Figure $1(\mathrm{~A})$ ) whereas staining with
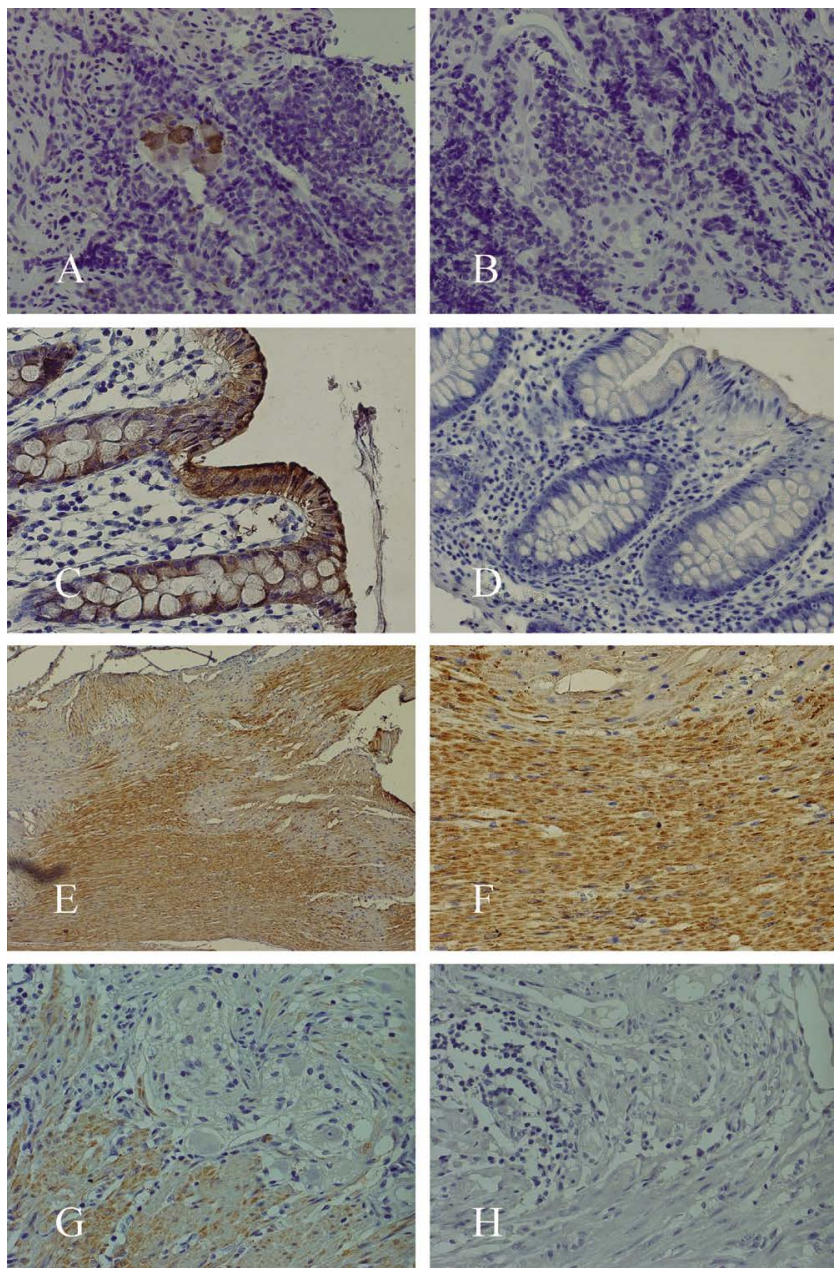

Figure 1. Crohn's disease. Immunoperoxidase staining of TI, colon biopsies and resected small bowel segment with enterovirus-specific mAb 5 D8/1 for enteroviral capsid protein 1 , with prior blocking with rabbit anti-CKb and anti-ATPase (see method section). (A) demonstrated viral protein in a rarely found granuloma of TI biopsy of case 3 (400x magnification), whereas (B) showed negative staining when performed with CMV mAb $(400 \mathrm{x})$. (C) showed positive staining in the sigmoid colon epithelium of patients 1 and 2 $(400 \mathrm{x})$, but not in biopsies of TI and other more inflamed areas of colon (400x, (D)). For patient 4, immunostaining demonstrated extensive enteroviral protein in the muscles of involved segment (IE 100x, (F) 400x). In an adjacent area, much less viral protein was seen in the muscles surrounding an intact myenteric plexus $((G), 400 x)$, although viral protein was not demonstrated in the muscles of uninvolved margins of TI and cecum $((\mathrm{H}), 400 \mathrm{x})$. 
CMV mAb showed negative result (Figure 1(B)). EV staining of her colon biopsies showed negative results (photo not shown). For patients 1 and 2, staining for EV VP1 showed positive staining in the sigmoid colon samples (Figure 1(C)), but not in biopsies of TI and other more inflamed areas of colon (Figure 1(D)). For patient 4, immunostaining demonstrated extensive enteroviral protein in themuscles of involved segment (Figure 1(E), Figure 1(F)). In an adjacent area, much less viral protein was seen in the muscles surrounding an intact myenteric plexus (Figure $1(\mathrm{G})$ ), although viral protein was not demonstrated in the muscles of uninvolved margins of TI and cecum (Figure $1(\mathrm{H})$ ). Similar findings were demonstrated in 3 other Crohn's disease patients who had resection of TI. Staining of the same sections with CMV antibody showed negative results (data not shown).

Overall, 8/10 of the patients had positive EV staining of colon epithelium (Table 1), whereas $3 / 78$ colon biopsy samples from screening colonoscopies of normal subjects were positive ( $\mathrm{p}<0.0001, \chi^{2}$ test with Yates correction). 9/10 TI biopsies or resected TI specimens showed no viral protein staining of the epithelium except specimen from case 3 demonstrating viral protein in the center of granuloma. 4/4 patients with resected terminal ileum showed muscle involvement in the diseased area but not in the normal TI or colon segments. Pre-incubation with 1:100 dilutions of polyclonal rabbit anti-CKb and anti-ATPase did not change the staining of the epithelium and muscles, and thereby excluded non-specific binding to these possible cross-reactive human mitochondrial epitopes (data not shown).

\section{Neutralizing antibody assay}

A modified E18 neutralization test using $\mathrm{CaCO}_{2}$ cells showed significantly elevated neutralizing antibody titers $(\geq 1: 80)$ in $8 / 8$ (100\%) of the Crohn's disease patient with available serum samples (Table 1), whereas only 11/85 (13\%) of patients with myalgic encephalomyelitis/chronic fatigue syndrome seen in the clinic during the same periods tested positive, $\left(\mathrm{P}<0.00001, \chi^{2}\right.$ test with Yates correction). However, the same serum samples failed to neutralize E18 in BGMK-DAF cells in two experiments (data not shown). Neutralizing antibody titers for CVB1, 2, 3, 4, 5, 6 and echovirus $6,7,9,11,30$ were elevated at $\geq 1: 80$ for $1 / 8,1 / 8,2 / 8,1 / 8,0 / 8,0 / 8,0 / 8,0 / 8,1 / 3,1 / 8,0 / 3$ patients, respectively (Table $1)$.

Enterovirus RNA was not detected in the paraffin sections of the 4 resected terminal ileum specimens.

\section{Discussion}

The small epidemic of CD patients and concurrent isolation of E18 in CSF specimens from patients with viral meningitis during the same periods provided the clues for this investigation. The cluster outbreak in a pair of identical twin teenagers, living in an area highly endemic for enterovirus infection due to frequently contaminated drinking water [10], was consistent with an environmen- 
tal factor for this disease. The finding of neutralizing antibody to E18 in all eight cases of $C D$ with available serum samples, as compared to $11 / 85$ control subjects living the same geographic area in the same periods supports the importance of E18 in CD cases. All the cases have pathologic evidence of EV protein in the biopsy or resected intestinal samples.

The inability to detect EV RNA in the inflamed colon was not unexpected due to the severe inflammatory response in the intestinal tissue specimens. VP 1 protein was not detected in multiple biopsies of the severely inflamed colon, but was more obvious in less inflamed areas. It is possible that the host inflammatory response interfered with viral RNA replication and viral protein synthesis resulting in false-negative results.

It was interesting that E18 was neutralized by serum samples from $\mathrm{CD}$ patients before incubating with $\mathrm{CaCO}_{2}$ cells, but not with BGMK-DAF cells. The mechanism for this discrepancy is unknown but E18 replicated at least $40-100$ times faster in BGMK-DAF cells than in $\mathrm{CaCO}_{2}$ cells, and could have overcome partial neutralization. Ironically, slow growth of $\mathrm{E} 18$ in $\mathrm{CaCO}_{2}$ cell, derived from colonic adenocarcinoma, may have more relevance to $\mathrm{CD}$, as compared to genetically-modified monkey kidney cells.

E18 may not be the only enterovirus associated with $\mathrm{CD}$, but the occurrence of small epidemics in 2004-2005, and finding positive E18 antibody in patients acquired disease 20 years earlier and in the recent past supported its importance. There are currently more than $65+$ serotypes and perhaps close to 140 genotypes, it is likely that other strains may play a role in this disease. However, in the studied patients with $\mathrm{CD}$, there were no consistently elevated antibody titers for 11 of the most common enteroviruses isolated in the past 30 years. Although patient $\# 5$ has significantly elevated antibody titers to CVB1 and CVB4, none of the other patients have similar findings. Heterotypic response to other enteroviruses may occur with the immune response to E18, but antibody raised in horses against E18 did not have a significant heterotypic reaction to other echoviruses, CVA1-24 and CVB1-6 or vice versa [14].

There is very little medical literature describing human E18 infection. The most commonly reported clinical diseases associated with E18 infections are gastroenteritis and aseptic meningitis, but paralysis, neonatal sepsis, and other manifestations also have been documented. E18 accounted for $2.7 \%$ of viral isolates with known serotypes in the CDC surveillance report. The virus has an epidemic pattern of circulation with increased levels of activity after prolonged quiescence. During the years 1970-2005, two periods of increased activity of E18 were observed (1986-1987 and 1995-2005). Activity reached its highest level in 2001. E18 appeared among the 15 most common enteroviruses during 18 of 36 years of the study period; it has never been the predominant enterovirus, but ranked second in 1987 and 2001. Children aged < 1 year were the most common age group for echovirus 18 detection, and CSF was the most common specimen. Death was reported for 1.8\% of E18 cases with known outcomes [1]. 
Besides infections of the stomach [8], enteroviruses can cause acute and chronic small bowel and colon infections [9]. Cluster outbreaks of intussusceptions [10] and small bowel obstruction and colonic volvulus, appendicitis in immunocompetent patients provided additional clues to the possible viral etiology [15], author's manuscript in preparation). Clustering outbreaks of Crohn's disease have been reported [16] [17]. Although the involvement of the myenteric plexus is common in Crohn's disease [5], infection of intestinal smooth muscles with proliferation, myofibroblastic transformation, hyper contraction and necrosis likely led to the transmural inflammation-a sine qua non of Crohn's disease [18]. Enteroviruses commonly infect skeletal and cardiac muscles and it can persist in these muscles in human and animal models of enterovirus infections [6] [11] [12].

A major issue with diagnosis of enterovirus infection in a patient with Crohn's disease is that terminal ileum mucosa rarely stained with $5 \mathrm{D} 8 / 1$ but deeper biopsies could not be safely obtained by colonoscopy. A full-thickness specimen, i.e. a resected small intestine, will be needed to find viral protein. The colon involvement tends to be patchy in nature, and could be missed without multiple biopsies of the inflamed areas and adjacent areas. Ironically, areas of more intense inflammation, showed minimal or no viral protein, but adjacent areas with less inflammation have more stainable viral protein (author's unpublished observation). The potential cross-reaction between enterovirus VP1 protein epitope and that of human mitochondrial proteins have been previously addressed by other studies [11] [12], and also in our laboratory using stomach biopsy samples [13]. Serotype-specific monoclonal antibody may have better sensitivity and specificity but it is not currently available. Significantly elevated titer of neutralizing antibody to E18 may be a useful diagnostic tool in patients with CD.

One of the weaknesses of these observations is that no other reagents are available for the diagnosis of E18 infection other than 5D8/1 monoclonal antibody, which has been showed to cross-react with other mitochondrial protein epitopes [11] [12]. The staining was performed with very low dilutions of this monoclonal antibody, and blocked with antibodies specific for the cross-reactive epitopes, creatine phosphokinase $\mathrm{b}$ and $\mathrm{Na}^{+} / \mathrm{K}^{+}$ATPase with identical results. Perhaps, type-specific monoclonal antibody will offer better staining of the tissues. Additional work on finding conserved enterovirus RNA sequences from tissue samples may add even stronger evidence for the role of enterovirus in this disease. Furthermore, larger cross-sectional studies of patient cohorts from different geographic areas will be needed to confirm the prevalence and importance of $\mathrm{E} 18$ in CD.

The mainstay of therapy for Crohn's disease is suppression of genetically-predisposed inflammatory responses [5]. If importance of E18 and other enteroviruses are confirmed by larger studies, specific antiviral drugs for enteroviruses may be investigated as an adjunctive therapy for Crohn's disease; and furthermore, vaccination against specific enteroviruses may potentially prevent a chronic, debilitating disease. 


\section{Acknowledgements}

We are grateful to Dr. John Blakey, Torrance Memorial Hospital pathologist, for his valuable suggestions for this investigation.

\section{Conflicts of Interest}

The authors declare no conflicts of interest regarding the publication of this paper.

\section{References}

[1] Khetsuriani, N., LaMonte-Fowlkes, A., Oberste, M. and Pallansch, M. (2006) Enterovirus Surveillance-United States, 1970-2005. MMWR Surveillance Summaries, 55, 1-20. https://doi.org/10.1037/e540562006-001

[2] Abramowsky, C.R. and Sorensen, R.U. (1988) Regional Enteritis-Like Enteropathy in a Patient with Agammaglobulinemia: Histologic and Immunocytologic Studies. Human Pathology, 19, 483-486. https://doi.org/10.1016/S0046-8177(88)80503-3

[3] Washington, K., Stenzel, T.T., Buckley, R.H. and Gottfried, M.R. (1996) Gastrointestinal Pathology in Patients with Common Variable Immunodeficiency and X-Linked Agammaglobulinemia. The American Journal of Surgical Pathology, 20, 1240-1252. https://doi.org/10.1097/00000478-199610000-00010

[4] Leparc, I., Aymard, M. and Fuchs, F. (1994) Acute, Chronic and Persistent Enterovirus and Poliovirus Infections: Detection of Viral Genome by Seminested PCR Amplification in Culture-Negative Samples. Molecular and Cellular Probes, 8, 487-495. https://doi.org/10.1006/mcpr.1994.1070

[5] Celier, C., Foray, S. and Hermine, O. (2000) Regional Enteritis Associated with Enterovirus in a Patient with X-Linked Agammaglobulinemia. The New England Journal of Medicine, 342, 1611-1612. https://doi.org/10.1056/NEJM200005253422113

[6] Nyström, N., Berg, T., Lundin, E., et al. (2013) Human Enterovirus Species b in Ileocecal Crohn's Disease. Clinical and Translational Gastroenterology, 4, e38. https://doi.org/10.1038/ctg.2013.7

[7] Nix, W.A., Oberste, M.S. and Pallansch, M.A. (2006) Sensitive, Seminested PCR Amplification of VP1 Sequences for Direct Identification of All Enterovirus Serotypes from Original Clinical Specimens. JCM, 44, 2698-2704. https://doi.org/10.1128/JCM.00542-06

[8] Chia, J. and Chia, A. (2008) Chronic Fatigue Syndrome Is Associated with Chronic Enterovirus Infection of the Stomach. Journal of Clinical Pathology, 61, 43-48. https://doi.org/10.1136/jcp.2007.050054

[9] Chia, J., Chia, A., Voeller, M., Lee, T. and Chang, R. (2010) Acute Enterovirus Infection Followed by Myalgic Encephalomyelitis/Chronic Fatigue Syndrome (ME/CFS) and Viral Persistence. Journal of Clinical Pathology, 63, 165-168. https://doi.org/10.1136/jcp.2009.070466

[10] Chia, A.Y. and Chia, J.K. (2009) Intestinal Intussusception in Adults Due to Acute Enterovirus Infection. Journal of Clinical Pathology, 62, 1026-1028. https://doi.org/10.1136/jcp.2008.063610

[11] Hansson, S., Korsgren, S., Ponten, F. and Korsgren, O. (2013) Enteroviruses and the Pathogenesis of Type 1 Diabetes Revisited: Cross-Reactive of Enterovirus Capsid 
Protein (VP1) Antibodies with Human Mitochondrial Proteins. The Journal of Pathology, 229, 719-728. https://doi.org/10.1002/path.4166

[12] Richardson, S., Leete, P., Dhayal, S., et al. (2014) Evaluation of the Fidelity of Immunostaining Obtained with Cloned 5D8/1, a Monoclonal Antibody Directed against the Enterovirus Capsid Protein, VP1, in Human Pancreas. Diabetologia, 57, 392-401. https://doi.org/10.1007/s00125-013-3094-7

[13] Chia, J., Chia, A., Wang, D. and El-Habbal, R. (2015) Functional Dyspepsia and Chronic Gastritis Associated with Enteroviruses. Open Journal of Gastroenterology, 5, 21-27. https://doi.org/10.4236/ojgas.2015.54005

[14] Melnick, J. and Hampil, B. (1970) WHO Collaborative Studies on Enterovirus Reference Antisera. 3rd Report. Bulletin of the World Health Organization, 42, 847-863.

[15] Alder, A.C., Fomby, T.B., Woodward, W.A., Haley, R.W., Sarosi, G. and Livingston, E.H. (2010) Association of Viral Infection and Appendicitis. The Archives of Surgery, 145, 63-71. https://doi.org/10.1001/archsurg.2009.250

[16] Allan, R.N., Pease, P. and Ibbotson, J.P. (1986) Clustering of Crohn's Disease in a Cotswold Village. Quarterly Journal of Medicine, 59, 473-476.

[17] Kruiningen, V. and Freda, B.J. (2001) A Clustering of Crohn's Disease in Mankato, Minnesota. Inflammatory Bowel Diseases, 7, 27-33. https://doi.org/10.1097/00054725-200102000-00004

[18] Dvorak, A., Osage, J., Monahan, R. and Dickersin, G.R. (1980) Crohn's Disease: Transmission Electron Microscopic Studies. III. Target Tissues. Proliferation of and Injury to Smooth Muscle and the Autonomic Nervous System. Human Pathology, 11, 620-634. https://doi.org/10.1016/S0046-8177(80)80073-6 